Western University

Scholarship@Western

Brain and Mind Institute Researchers'

Publications

Brain and Mind Institute

7-1-2014

\title{
Self-injurious behaviours are associated with alterations in the somatosensory system in children with autism spectrum disorder.
}

\author{
Emma G Duerden \\ Department of Diagnostic Imaging, Hospital for Sick Children, 555 University Avenue, Toronto, ON M5G \\ $1 X 8$, Canada \& Program in Neurosciences and Mental Health, Hospital for Sick Children, Toronto, ON, \\ Canada, emma.duerden@sickkids.ca \\ Dallas Card \\ Department of Diagnostic Imaging, Hospital for Sick Children, 555 University Avenue, Toronto, ON M5G \\ $1 X 8$, Canada \\ S Wendy Roberts \\ Department of Diagnostic Imaging, Hospital for Sick Children, 555 University Avenue, Toronto, ON M5G \\ $1 \times 8$, Canada \\ Kathleen M Mak-Fan \\ Department of Diagnostic Imaging, Hospital for Sick Children, 555 University Avenue, Toronto, ON M5G \\ $1 X 8$, Canada \& Department of Psychology, University of Toronto, Toronto, ON, Canada \\ M Mallar Chakravarty \\ Kimel Family Translational Imaging-Genetics Research Laboratory, Research Imaging Centre, Centre for \\ Addiction and Mental Health, Toronto, ON, Canada \& Department of Psychiatry and Institute of \\ Biomaterials and Biomedical Engineering, University of Toronto, Toronto, ON, Canada \\ Follow this and additional works at: https://ir.lib.uwo.ca/brainpub
}

Part of the Neurosciences Commons, and the Psychology Commons See next page for additional authors

Citation of this paper:

Duerden, Emma G; Card, Dallas; Roberts, S Wendy; Mak-Fan, Kathleen M; Chakravarty, M Mallar; Lerch, Jason P; and Taylor, Margot J, "Self-injurious behaviours are associated with alterations in the somatosensory system in children with autism spectrum disorder." (2014). Brain and Mind Institute Researchers' Publications. 169.

https://ir.lib.uwo.ca/brainpub/169 


\section{Authors}

Emma G Duerden, Dallas Card, S Wendy Roberts, Kathleen M Mak-Fan, M Mallar Chakravarty, Jason P Lerch, and Margot J Taylor 


\title{
Self-injurious behaviours are associated with alterations in the somatosensory system in children with autism spectrum disorder
}

\author{
Emma G. Duerden - Dallas Card · S. Wendy Roberts • \\ Kathleen M. Mak-Fan · M. Mallar Chakravarty • \\ Jason P. Lerch $\cdot$ Margot J. Taylor
}

Received: 10 December 2012/Accepted: 19 April 2013/Published online: 5 May 2013

(C) Springer-Verlag Berlin Heidelberg 2013

\begin{abstract}
Children with autism spectrum disorder (ASD) frequently engage in self-injurious behaviours, often in the absence of reporting pain. Previous research suggests that altered pain sensitivity and repeated exposure to noxious stimuli are associated with morphological changes in somatosensory and limbic cortices. Further evidence from postmortem studies with self-injurious adults has indicated alterations in the structure and organization of the temporal lobes; however, the effect of self-injurious behaviour on
\end{abstract}

E. G. Duerden $(\bowtie) \cdot$ D. Card $\cdot$ S. W. Roberts

K. M. Mak-Fan · M. J. Taylor

Department of Diagnostic Imaging, Hospital for Sick Children,

555 University Avenue, Toronto, ON M5G 1X8, Canada

e-mail: emma.duerden@sickkids.ca

E. G. Duerden · J. P. Lerch · M. J. Taylor

Program in Neurosciences and Mental Health, Hospital for Sick

Children, Toronto, ON, Canada

K. M. Mak-Fan · M. J. Taylor

Department of Psychology, University of Toronto, Toronto, ON, Canada

M. M. Chakravarty

Kimel Family Translational Imaging-Genetics Research

Laboratory, Research Imaging Centre, Centre for Addiction and Mental Health, Toronto, ON, Canada

M. M. Chakravarty

Department of Psychiatry and Institute of Biomaterials and

Biomedical Engineering, University of Toronto,

Toronto, ON, Canada

J. P. Lerch

Department of Medical Biophysics, University of Toronto,

Toronto, ON, Canada cortical development in children with ASD has not yet been determined. Thirty children and adolescents (mean age $=10.6 \pm 2.5$ years; range 7-15 years; 29 males) with a clinical diagnosis of ASD and 30 typically developing children $(N=30$, mean age $=10.7 \pm 2.5$ years; range 7-15 years, 26 males) underwent T1-weighted magnetic resonance and diffusion tensor imaging. No between-group differences were seen in cerebral volume, surface area or cortical thickness. Within the ASD group, self-injury scores negatively correlated with thickness in the right superior parietal lobule $t=6.3, p<0.0001$, bilateral primary somatosensory cortices (SI) (right: $t=4.4, p=0.02$; left: $t=4.48, p=0.004$ ) and the volume of the left ventroposterior (VP) nucleus of the thalamus $(r=-0.52$, $p=0.008$ ). Based on these findings, we performed an atlas-based region-of-interest diffusion tensor imaging analysis between SI and the VP nucleus and found that children who engaged in self-injury had significantly lower fractional anisotropy $(r=-0.4, p=0.04)$ and higher mean diffusivity ( $r=0.5, p=0.03$ ) values in the territory of the left posterior limb of the internal capsule. Additionally, greater incidence of self-injury was associated with increased radial diffusivity values in bilateral posterior limbs of the internal capsule (left: $r=0.5, p=0.02$; right: $r=0.5, p=0.009$ ) and corona radiata (left: $r=0.6, p=0.005$; right: $r=0.5, p=0.009$ ). Results indicate that self-injury is related to alterations in somatosensory cortical and subcortical regions and their supporting white-matter pathways. Findings could reflect use-dependent plasticity in the somatosensory system or disrupted brain development that could serve as a risk marker for self-injury.

Keywords Autism - Spectrum disorder - Injury · Grey matter $\cdot$ White matter Pain 


\section{Introduction}

Children and adolescents with autism spectrum disorder (ASD) often perform harmful acts directed towards themselves (Baghdadli et al. 2003; Duerden et al. 2012; Militerni et al. 2002). Self-injurious behaviours are sometimes repetitive and can be rhythmic. These behaviours range from mild hair pulling to much more severe actions such as head banging or autoextraction of teeth (Armstrong and Matt 1999; Medina et al. 2003; Ross-Russell and Sloan 2005), and are often performed in the absence of reporting pain. Selfinjury may be associated with alterations in brain regions that process both sensory-discriminative and affective components of pain and may reflect atypical somatosensory processing commonly seen in this population (Tordjman et al. 2009). However, the effects of self-injury on the development of grey and white matter have yet to be determined.

Repeated exposure to noxious stimuli and altered pain sensitivity has been associated with morphological changes in brain regions that process somatosensation and pain affect in healthy adults (Grant et al. 2010; Teutsch et al. 2008). Zen Buddhists, who regularly sit in postures that become painful, had increased thickness in the primary somatosensory cortex (SI) associated with hours of practice, had reduced pain sensitivity and showed increased thickness in brain regions that mediate pain intensity (secondary somatosensory cortex, SII), pain affect [anterior cingulate cortex (ACC)], and pain modulation (parahippocampal gyrus) (Grant et al. 2010). Additionally, healthy participants who received noxious stimuli repeatedly over an 8-day period had reduced pain sensitivity and increased grey matter density in the ACC and SI contralateral to the stimuli (Teutsch et al. 2008).

Neuropathological studies of self-injurious adults with ASD have demonstrated changes in the structure and organization of neuronal tissues, particularly in the temporal lobes (Hof et al. 1991; Wegiel et al. 2010). However, these studies included small samples, and investigation of the effects of self-injury on brain structure both in vivo and in larger samples of individuals with ASD is warranted.

Several brain regions may be altered in individuals with ASD who self-injure including the thalamus. The thalamus actively filters sensory information to the cortex. Several reports have theorized that sensory abnormalities demonstrated by individuals with ASD are associated with disrupted thalamocortical processing (Baranek 2002; Hardan et al. 2006a; Tsatsanis et al. 2003).

Previous postmortem studies on self-injurious individuals with ASD primarily focussed on grey matter changes in the cortex and cerebellum; however, alterations in whitematter fibre pathways are also associated with repetitive movements. Thakkar et al. (2008) correlated diffusion tensor imaging (DTI) measures of fractional anisotropy
(FA, measure of tensor directionality that is generally believed to reflect myelination and axonal density) with parental ratings of repetitive behaviours. Lower FA in the ACC was associated with increased repetitive behaviour. Additionally, these authors noted reduced FA in the frontal cortex, rostral ACC and paracentral lobule/SI. Atypicalities in white-matter fibres, reflected by alterations in FA, have also been reported in children with repetitive-brain injury (Lipton et al. 2012), which may be found in a subset of children with ASD who self-injure.

Presently, little is known concerning the effects of selfinjury on the development of cortical and subcortical structures involved in somatosensory processing and the underlying white-matter pathways in children with ASD. In the current study, we tested the hypothesis that self-injurious behaviours would be associated with changes in SI, medial temporal cortices, cingulate and thalamus, as well as the thalamocortical pathways.

\section{Methods}

\section{Participants}

The full ASD cohort included 33 children and adolescents (mean age $=10.7 \pm 2.5$ years; range $7-15$ years; 29 males; 28 right-handed, two left-handed, three handedness data not available) with a clinical diagnosis of ASD (Table 1). The ASD diagnosis was confirmed with the Autism Diagnostic Observation Schedule-Generic (ADOS-G) (Lord et al. 2000) and the Autism Diagnostic Interview-Revised (ADI-R) (Le Couteur et al. 2003). Diagnostic assessments were administered by a developmental paediatrician or a clinical psychologist. All personnel had achieved research-level reliability with the University of Michigan Autism and Communication Disorders Center. All ASD participants were healthy, verbal and had the intellectual capacity to undergo the scanning session successfully (IQ $=104.1 \pm 18.3$ ). None of the participants was taking any medication at the time of study. The ASD cohort was recruited through fliers at the Autism Research Unit at the Hospital for Sick Children, Toronto, Canada.

The comparison cohort was a group of age-matched typically developing (TD) children and adolescents $(N=30$, mean age $=10.7 \pm 2.5$ years; range $7-15$ years, 26 males). The majority of the children were right-handed $(N=22$, left-handed $=2$; not available $=6)$, and had a mean IQ that was higher than the ASD population $(113.6 \pm 13.7$; Table 1$)$. TD children were not included in the study if they had a family history of ASD, neurological/ psychiatric disease, or medication usage. The TD children were recruited through fliers in the hospital or at local schools and announcements in the Hospital for Sick Children newsletter. 
Table 1 Participant demographics

\begin{tabular}{llll}
\hline & ASD & TD & $\begin{array}{l}\text { Between-group } \\
\text { comparisons }\end{array}$ \\
\hline$N$ & 33 & 30 & \\
Sex (male) & 29 & 26 & $\chi^{2}=0.02, p=0.9$ \\
Handedness & $28 \mathrm{R} 2 \mathrm{~L}$ & 22R 2L & $\chi^{2}=0.05, p=0.8$ \\
Age & $10.7 \pm 2.5$ & $10.7 \pm 2.5$ & $T=0.35, p=0.97$ \\
IQ & $104 \pm 18.3$ & $113.6 \pm 13.7$ & $T=2.2, p=0.03$ \\
\hline
\end{tabular}

The sex and handedness data were tested for independence using Pearson's Chi square. Between-group age and IQ differences were assessed using an independent $t$ test. The alpha level was set at 0.05 for all statistical tests. Handedness information was not available in three of the ASD (Autism spectrum disorder) and six of the TD (typically developing) children

The study was approved by the research ethics board at the Hospital for Sick Children. Written informed consent was obtained from parents and adolescents, and informed assent from the children.

\section{Self-injurious behaviour assessment}

Parents of children with ASD completed the Repetitive Behaviour Scale-Revised (RBS-R) (Bodfish et al. 1999). This measure was not given to the parents of TD children. The RBS-R is a standardized questionnaire that has been independently validated in individuals with ASD (Lam 2007). The questionnaire includes eight statements on recent (within 1 month) self-injurious behaviour and each item is rated on a four-point Likert scale ranging from low (0) to high (3) severity of self-injury (maximum score $=24)$. The items from the RBS-R include: (1) Hits self with body part; (2) Hits self against object; (3) Hits self with object; (4) Bites self; (5) Pulls (hair or skin); (6) Rubs or scratches self; (7) Inserts finger or object: eye poking, ear poking; (8) Skin picking. High scores reflect greater incidence and/or severity of self-injury.

Parents were also questioned as to the location, frequency, and duration of any reported self-injurious behaviour. Additionally, parents were asked to rate their child's pain reactivity in relation to TD children (low, typical, high).

\section{Magnetic resonance imaging}

High-resolution anatomical images were acquired using a 1.5 T GE Signa Excite III HD 12.0 MRI system (General Electric Medical Systems, Milwaukee, Wisconsin) with an 8-channel head coil. A T1-weighted 3D fast spoiled gradient echo (FSPGR) sequence $(\mathrm{TR}=9 \mathrm{~ms}$; $\mathrm{TE}=4.2 \mathrm{~ms}$ ) was used to generate $1101.5 \mathrm{~mm}$-thick axial slices $(256 \times 192$ matrix, $24 \mathrm{~cm}$ field of view). This sequence was followed by a single-shot echo planar DTI scan using a spin echo sequence
$(\mathrm{TR} / \mathrm{TE}=10,000 / 88.7 \mathrm{~ms}$; motion probing gradient in 35 diffusion-encoding directions $\left[b\right.$ value $\left.=1,000 \mathrm{~s} / \mathrm{mm}^{2}\right]$ with 3 non-diffusion-weighted images $\left[b\right.$ value $\left.=0 \mathrm{~s} / \mathrm{mm}^{2}\right]$; $256 \times 256$ matrix; $0.9375 \times 0.9375 \mathrm{~mm}$ in-plane resolution; $3 \mathrm{~mm}$ slice thickness).

Cerebral volume, surface area and cortical thickness measurements and analysis

Anatomical MRIs were preprocessed using a standard protocol using the automated corticometric iterative vertexbased estimation of thickness (CIVET) image-processing pipeline (Lerch et al. 2005). The methods have been described in detail elsewhere (Lerch et al. 2005). In brief, the T1-weighted images underwent correction for nonuniformity artefacts (Sled et al. 1998), were skull stripped (Smith 2002) and aligned to a common image space (Collins et al. 1994). Brain tissue was classified into white matter (WM), grey matter (GM) and cerebrospinal fluid (CSF) (Tohka et al. 2004; Zijdenbos et al. 2002). For each image, GM, WM, and CSF volumes were calculated for the whole brain. Additionally, the surface areas of 16 regions (bilateral parietal, occipital, frontal, temporal lobes, isthmus of the cingulate gyrus, parahippocampal gyrus, cingulate gyrus and the insula) were automatically calculated.

Group differences in GM and WM and total brain volume were assessed using univariate analyses, controlling for sex, age, and handedness. The between-group differences in GM and WM volumes were also assessed controlling for total cerebral volume.

Between-group differences in surface area at a lobar/ regional level were investigated using a MANCOVA, controlling for age, sex and handedness. The anatomical delineations of the lobes and regions-of interest have been described in detail elsewhere (Lax et al. 2013).

For the cortical thickness analysis, the inner and outer cortical surfaces with 81,924 vertices each were extracted using partial-volume-effect classification. Cortical thickness was determined in native space using the distance between the outer and pial surfaces at each vertex. Images were smoothed (20 mm (Chung et al. 2001)) and non-linearly registered to a template surface (Boucher et al. 2009; Lyttelton et al. 2007).

Cortical thickness analyses were performed using the Matlab-based program SurfStat (Worsley et al. 2009). A series of linear models were performed to assess between group differences (ASD vs. TD) and the relation between self-injury and changes in cortical morphology. For the latter analyses, cortical thickness was regressed on selfinjury scores. All analyses controlled for age and sex. Handedness was also controlled for in the analyses as this variable has been shown to influence cortical thickness (Haller et al. 2009; Hamilton et al. 2007). 
Based on a priori hypotheses, regions-of-interest (ROI) were manually drawn bilaterally in SI, the entire cingulate cortices and medial temporal cortices based on anatomical landmarks. Whole brain and ROI data were cluster corrected for multiple comparisons using Random Field Theory (RFT; $p<0.05$ ) (Worsley et al. 1996).

\section{Subcortical measurements and analysis}

The entire thalamus and Hirai and Jones thalamic subdivisions were automatically segmented by customizing a high-resolution subcortical atlas derived from serial histological data (Chakravarty et al. 2006) using a ROI nonlinear registration procedure (Chakravarty et al. 2008). The subcortical segmentation routine has been validated using manual gold-standard segmentations, against other automated segmentation techniques, and intraoperative electrophysiological recordings in humans undergoing stereotactic neurosurgery (Chakravarty et al. 2008, 2009). No a priori hypotheses were formulated concerning the association between self-injury and differences in thalamic volumes. Therefore, an exploratory partial correlation analysis (two-tailed), controlling for age, sex and handedness, was used to assess the relation between the volume of each thalamic nuclei and self-injury scores. Significance values were set at $p<0.05$.

\section{Diffusion tensor imaging analysis}

DTI analyses were performed using both Camino (Cook et al. 2006) and the FMRIB software library (FSL, http://www.fmrib.ox.ac.uk/fsl/). Images underwent motionand eddy-current effect corrections. Diffusion-weighted volumes were linearly registered to one non-diffusion weighted volume using affine transformations (FLIRT) (Smith et al. 2004), and outlier rejection was accomplished with the RESTORE algorithm (Chang et al. 2005). Estimated diffusion tensor data were masked to include only the brain (Smith 2002). To measure overall diffusivity, a diffusion tensor model was fit to the data at each voxel and used to calculate voxel-wise FA and mean diffusivity (MD) values using Camino. Directional diffusivities including axial diffusivity (diffusivity along the axon; $A D=$ first eigenvalue, $\lambda 1$ ) and radial diffusivity (diffusivity perpendicular to the axon; RD = average of second and third eigenvalues, $\lambda 2, \lambda 3$ ) were also calculated. An increase in axonal number or size (Hildebrand and Waxman 1984; Song et al. 2002) and/or reduced interaxonal space (Qiu et al. 2008; Suzuki et al. 2003; Takahashi et al. 2000) will result in reduced diffusivity along the axon, reflected in a decrease in AD. Enhanced myelination will lead to reduced permeability of the myelin sheath and be reflected in a decrease in RD (Qiu et al. 2008; Suzuki et al. 2003).
The tract-based spatial statistics (TBSS) pipeline (Smith et al. 2006) was used to register the FA volumes to a standard MNI template using non-linear registration (FNIRT), and to generate a white-matter skeleton running through the centre of all major white-matter tracts in the brain. This skeleton was masked using parts of the JHU ICBM-DTI 81 white-matter atlas (37, Harvard-Oxford cortical structural atlas, for details see http://www.fmrib.ox.ac.uk/fsl/data/atlas-descriptions.html) to isolate the cingulum bundle, posterior limb of the internal capsule (PLIC) and corona radiata as these are the main whitematter fibre pathways that connect the cortical and subcortical regions-of-interest.

The non-linear transformations calculated using the FA images were then applied to the $\mathrm{MD}, \mathrm{AD}$, and $\mathrm{RD}$ volumes to bring them into alignment with the template. Randomize (FSL) was used to perform voxel-wise statistics on the FA, $\mathrm{MD}, \mathrm{AD}$, and $\mathrm{RD}$ volumes for the voxels in the masked white-matter skeleton, including cluster-size thresholding at a level of $p<0.05$ and correction for multiple comparisons across space. Using this method, these four metrics were compared voxel-wise between groups and correlated with self-injury scores in the ASD group. Finally, the average FA, MD, AD, and RD values were also extracted for the entire masked white-matter skeleton.

\section{Results}

Self-injurious behaviour assessment

The RBS-R was administered to the parents of 29 children and adolescents with ASD. Scores ranged from 0 (no selfinjury present in the last month) to 6 (scoring on several items). High incidence of self-injury (scores $<2$ ) was reported in 12 of the children (mean $=2.31, \mathrm{SD}=1.4$ ). The 17 remaining children demonstrated low (score of 1) or no self-injury.

Twelve parents provided details on the location, duration and frequency of their child's self-injury. The locations of the injuries were often over multiple sites or covered large areas of the body. Facial injuries were present in four children, hand and arm injuries were performed by 4 children, and a leg injury was present in one child. The duration of the injuries ranged from 6 months to over 100 months. Half (6) of these children engaged in self-injury daily. Of the children who self-injured, seven were reported by their parents to have low pain reactivity, four had typical and three were rated to have high pain reactivity.

As few numbers of the parents provided information on the duration and frequency of self-injury and their child's pain reactivity, only the data from the RBS-R were used for the subsequent brain imaging analyses. 
Imaging results

T1-weighted anatomical images were successfully acquired in 58 participants ( $28 \mathrm{ASD}, 30 \mathrm{TD}$ ). The mean age of these participants with ASD was 10.5 ( $\mathrm{SD}=2.4,25$ males, 3 females; handedness: 24 right, 2 left, 2 data not available). In the ASD sample only, self-injury scores were acquired in all participants.

DTI data were acquired in 61 participants (31 ASD, 30 TD). The self-injury scores were available in 27 of these participants, and the data from one participant were removed due to excessive head motion (total ASD sample: 23 males, 3 females, mean age (years) $=10.7, \mathrm{SD}=2.6$; handedness: 24 right, 2 left).

\section{Between-groups (ASD vs. TD) analyses}

Total cerebral volume Total cerebral volume was not significantly different between the two groups $(F=0.23$, $p=0.87)$. Grey $(F=0.04, p=0.85)$ and white matter $(F=0.002, p=0.96)$ also did not vary between groups $(F=0.4, p=0.96)$. These results were maintained when controlling for total cerebral volume (GM: $F=0.01$, $p=0.92$; WM: $F=0.14, p=0.71)$ and IQ $(F=0.2$, $p=0.8$ ).

Cortical grey matter volume was not significantly different in the ASD group $\left(686 \pm 76 \mathrm{~cm}^{3}\right)$ compared to the TD group $\left(686 \pm 71 \mathrm{~cm}^{3}\right)$. Similar findings were found for white-matter volume (ASD: $495 \pm 42 \mathrm{~cm}^{3}$; TD: $494 \pm 61 \mathrm{~cm}^{3}$ ).

Surface area No main group-effects were seen in the surface area data for the 16 regions-of-interest (bilateral parietal, occipital, frontal, temporal lobes, isthmus of the cingulate gyrus, parahippocampal gyrus, cingulate gyrus and the insula; $F=1.3, p=0.26$ ); the results were maintained when controlling for IQ $(F=1.63, p=0.1)$.

Cortical thickness No significant increases or decreases in cortical thickness were apparent in the between-group analysis (TD vs. ASD). However, the ASD group showed trends towards an increase in thickness in the right paracentral lobule $(t=3.52, \quad x=78 \quad y=-7 \quad z=6264$, $p>0.05$, corrected) and the left middle frontal gyrus $(t=3.2, x=-31 y=21 z=47, p>0.05$, corrected). No significant differences were seen when controlling for IQ.

Subcortical volumes A significant main effect of group was found when comparing the volumes of the thalamic nuclei between the TD and ASD children $(F=16.06$, $p<0.0001)$. Results were maintained when controlling for total cerebral volume (TCV; $F=17.01, p<0.0001)$ and IQ $(F=14.52, p<0.0001)$. The results were driven by several nuclei that were significantly larger in the ASD group (bilateral lateral and medial geniculate, anterior, centromedian, ventroanterior, ventrolateral, and ventroposterior nuclei); only the right lateral dorsal nucleus was larger in controls.

DTI measures No significant main effects of group $(F=0.7, p=0.7)$ were seen when comparing mean FA, $\mathrm{MD}, \mathrm{AD}$, and $\mathrm{RD}$ values from the masked white-matter skeleton between the children with and without ASD; IQ did not affect these results $(F=0.4, p=0.95)$.

\section{Within-group (ASD) analyses}

Cortical thickness analysis Within the ASD sample $(n=28)$, based on a search of the entire cortical grey matter, self-injury scores were negatively correlated with thickness in the right superior parietal (SPL) and right SI $(t=6.3, p<0.0001 ; p<0.05$ corrected for multiple comparisons using RFT; Table 2; Fig. 1). A directed search in right SI revealed a similar significant negative correlation in this region $(t=4.4, p=0.02$, corrected) and also in left SI $(t=4.48, p=0.004$, corrected; Fig. 1). Similar results were seen when IQ was controlled (right SPL $t=6.4, p<0.0001$; right SI $t=4.4, p=0.02$; left SI $t=3.9, p=0.004$ ).

The participants' self-injury scores ranged widely $(0-6)$ and therefore the data from participants with highest scores may have unduly influenced the results. Subsequent post hoc analyses were performed with the data separated into groups based on the RBS-R scores. Participants with a score of 0 or $1(n=16)$ were classified as having infrequent or low self-injury and those with scores of 2 and above were classed as frequent or a higher incidence of self-injury $(n=12)$. A contrast between the infrequent (low) vs. more prevalent (higher incidence) self-injury groups (controlling for age, sex, and handedness) revealed similar results as the global-cortical search (right SPL: $t=5.53, p=0.0005$; right SI: $t=4.53, p=0.004$; $p<0.05$ corrected for multiple comparisons using RFT) and directed searches in the ROI (right SI: $t=4.53$, $p=0.004$; left SI: $t=3.89, p=0.009$, corrected).

The thickness values from left and right SI were extracted based on the between self-injury group contrast and a significant main effect was found for group (controlling for age, sex and handedness; right SI: $F=5.24$, $d f=1,22, \quad p=0.032 ; \quad$ left $\quad$ SI: $F=6.94, \quad d f=1,22$, $p=0.015$, corrected), with thinner cortex in the group with greater incidence of self-injury. The mean cortical thickness values and $95 \%$ confidence intervals for left and right SI in the infrequent (low) and greater incidence (high) groups are displayed in Fig. 2. 
Table 2 Self-injury is associated with thinner cortex in somatosensory brain areas cortical thickness data were regressed against selfinjury scores (converted to negative values to produce positive results), controlling for age, sex, and handedness. Coordinates are in the space of the Montreal Neurological Institute (MNI; Collins et al. 1994)

\begin{tabular}{lcccccc}
\hline ASD within group analyses (correlation with self-injury scores) & & & & \\
\hline Brain region & Side & $x$ & $y$ & $z$ & $P$ & $t$ \\
\hline Global Search & & & & & \\
$\quad$ Superior parietal lobule & Right & 11 & -51 & 74 & $<0.0001$ & 6.30 \\
$\begin{array}{l}\text { Directed search } \\
\text { Primary somatosensory cortex }\end{array}$ & Right & 11 & -45 & 75 & 0.02 & 4.4 \\
& Left & -56 & -18 & 53 & 0.004 & 4.48 \\
\end{tabular}

Data were corrected for multiple comparisons using Random Field Theory $(p<0.05)$

\section{Global grey-matter search}
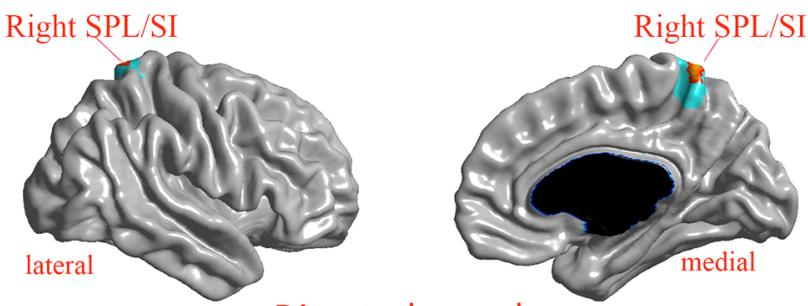

Directed search
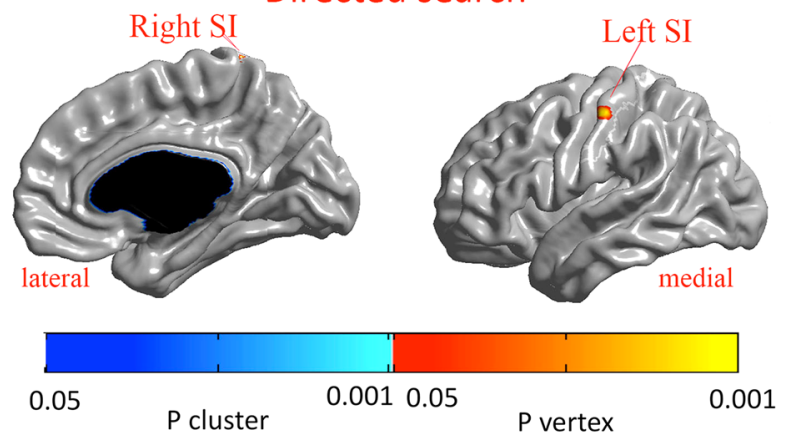

Fig. 1 Top Entire grey matter, vertex-based cortical thickness analysis (corrected $p$ map): corrected $p$ values demonstrating regions significantly decreased in relation to self-injury scores, controlling for age, sex and handedness and corrected for multiple comparisons using Random Field Theory $(p<0.05)$. Children with high self-injury scores had decreased thickness in the right superior parietal lobule (SPL)/primary somatosensory cortex (SI). Bottom Directed search in SI. Increased self-injury was associated with decreased thickness in SI. The colour bar indicates corrected $p$ values for significant peaks at the cluster- (right; light blue-dark blue) and vertex-levels (left; redorange)

\section{Subcortical volumetry}

Within the ASD group $(N=28)$, of the thalamic nuclei, only the volume of the ventroposterior (VP) nucleus was significantly negatively correlated with self-injury scores $(r=-0.52, p=0.008)$; these significant results were maintained when controlling for IQ $(r=-0.58$, $p=0.003)$. These analyses were largely exploratory and

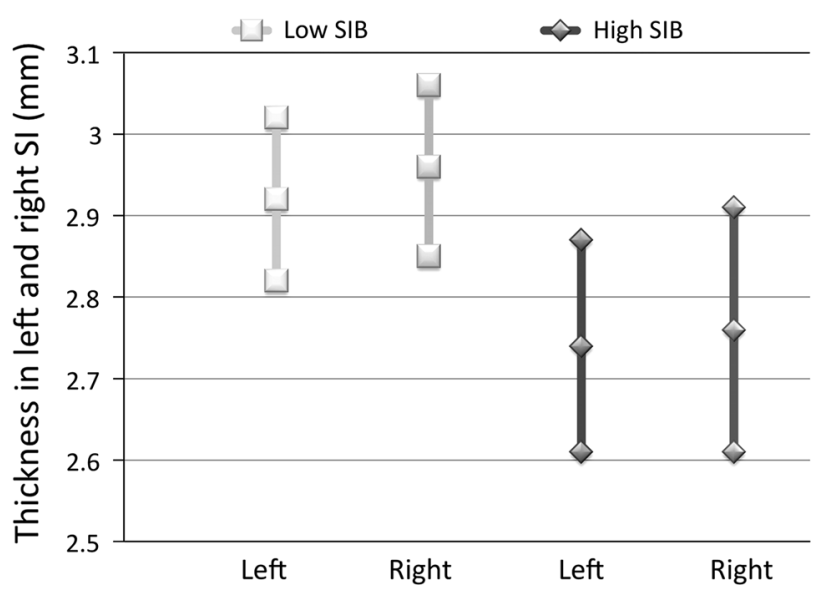

Fig. 2 Mean cortical thickness values from the left and right primary somatosensory cortices (SI) in the children with no or low self-injury (left) in comparison to those who had greater incidence and/or severity of self-injurious behaviours. Results indicate that those with high self-injury scores have thinner cortical thickness in SI. Error bars $=95 \%$ confidence interval of the mean cortical thickness

the association of self-injury scores with that of the volumes of all thalamic nuclei was assessed. Given the large number of nuclei, this result would not have survived an adjustment for multiple comparisons. However, these analyses were conducted to formulate a directed hypothesis, and a subsequent search in the left VP nucleus was conducted in a post hoc analysis.

A between self-injury group (low/no self-injurious behaviour vs. self-injurious behaviour) univariate analyses revealed that children with greater incidence (higher) selfinjury scores had significantly smaller left VP nuclei in comparison to children who had no or very low self-injury scores $(F=6.52, p=0.018$; Fig. 3$)$.

\section{DTI measures}

The data from 26 individuals were included in these analyses. A significant negative correlation of self-injury 


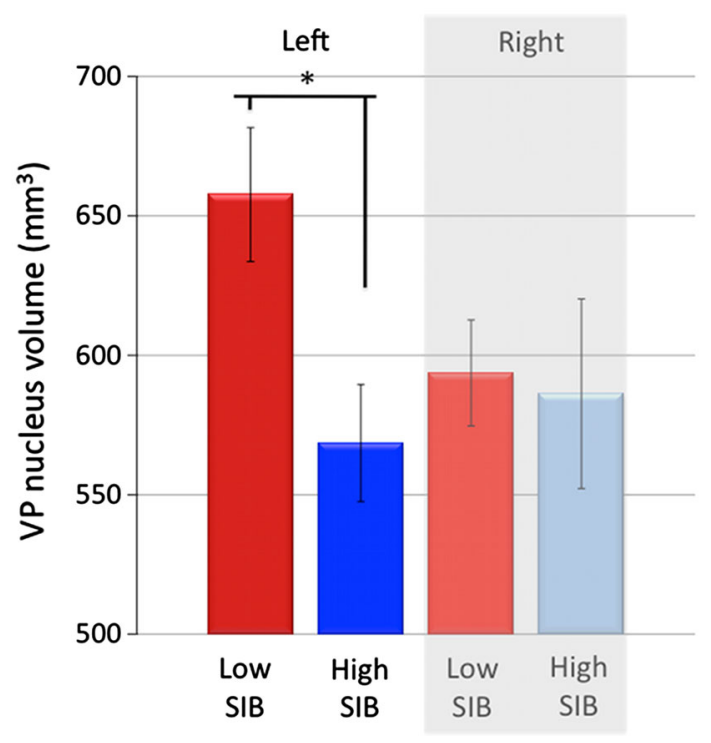

Fig. 3 Mean thalamic volume in the left and right ventroposterior nuclei for children with no/low (red) and high (blue) incidence of self-injurious behaviour. Asterisk significant at $p<0.05$

scores and FA was found in the territory of the left PLIC $(r=-0.4, p=0.04)$. Additionally, significant positive correlations among self-injury scores and $\mathrm{MD}(r=0.5$, $p=0.03$ ) were found in this region. Self-injury scores also positively correlated with RD values in the bilateral posterior limbs of the internal capsule (left: $r=0.5, p=0.02$; right: $r=0.5, p=0.009$ ) and corona radiata (left: $r=0.6, p=0.005$; right: $r=0.5, p=0.009$ ). None of these results was impacted when controlled for IQ (left PLIC: FA $r=-0.4, p=0.045$; MD $r=0.5, p=0.03$ $\mathrm{RD} r=0.5, p=0.03$; right PLIC: RD $r=0.5, p=0.01$; left corona radiata $\mathrm{RD} r=0.5, p=0.02$; right corona radiata $\mathrm{RD} r=0.6, p=0.009$ ).

A subsequent between self-injury groups (infrequent $n=12$; greater incidence $n=14$ ) analysis revealed a significant main effect for group $(F=3.3, p=0.03)$. However, only trends towards decreased FA $(F=4.06$, $p=0.057)$ were seen in the left PLIC in children with greater self-injury scores and increased $\mathrm{RD}$ in the right corona radiata $(F=3.99, p=0.059)$.

A slightly greater number of individuals with ASD had low or no previous incidence of self-injury in comparison to those who showed higher incidence. The within-group analyses were repeated using non-parametric statistics and showed similar results.

\section{Discussion}

The influence of self-injury on cortical and subcortical grey matter and white matter was assessed in children and adolescents with ASD. Greater self-injury scores were associated with thinner cortex in the SPL and bilateral SI. Furthermore, decreased volume of the left primary somatosensory relay nucleus (VP nucleus) of the thalamus was also found to be associated with greater incidence of self-injury. Lastly, lower FA and higher MD values were found in the left PLIC in children who had greater incidence of self-injury; this white-matter pathway contains the afferent fibres from the VP nucleus to SI. Also greater incidence of self-injury was positively correlated with RD values in the bilateral PLIC and corona radiata. Findings indicate that repetitive injury is associated with alterations in the somatosensory system. These preliminary results may be reflective of use-dependent plasticity as a result of noxious-stimulus induced changes in the somatosensory system caused by repetitive bodily harm. Alternatively, children who self-injure may have pre-existing abnormalities in the somatosensory system due to altered development in these areas. Longitudinal studies in children who self-injure are needed to determine which of these alternatives best explains these effects.

\section{Cortical grey and white matter in ASD and TD children}

In the current study, measures of total cerebral volume, GM, WM, surface area, and cortical thickness did not differ between the ASD and TD groups. This is somewhat inconsistent with previous findings, as others have reported differences in total cerebral volume, GM and WM. However, these results were more pronounced in young children (Aylward et al. 2002; Carper and Courchesne 2005; Carper et al. 2002; Courchesne 2004; Hardan et al. 2001) and the lack of difference in brain volume may be explained by the large age range of the participants, and that their mean age was over 10 years.

Previous studies examining indicators of alterations in surface area included analysis of gyrification indices, sulcal location and depth have indicated atypicalities in children with ASD (Hardan et al. 2004; Nordahl et al. 2007) or were based on a ROI analysis (Doyle-Thomas et al. 2012). As the results of the current study were based on a whole brain analysis, the lack of alterations in surface area may be reflective of differences in analytic methods.

Both decreased (Hadjikhani et al. 2006; Scheel et al. 2011; Wallace et al. 2010) and increased (Hyde et al. 2010) cortical thicknesses have been reported in adults with ASD in comparison to TD individuals. Few studies have examined cortical thickness changes in children and adolescents with ASD. We have found steeper decreases in reported decreased thickness with age in the frontal and parietal lobes for the ASD group (Mak-Fan et al. 2012), such that thickness was greater in the younger ages (under 10 years of age) and tended to be thinner in the mid teen-aged years, 
consistent with studies of either older or younger children (Hardan et al. 2006b; Misaki et al. 2012). Others have reported decreased thickness in specific regions of the temporal and occipital lobes (Scheel et al. 2011; Wallace et al. 2010) in adolescents and young adults, with other brain regions showing little developmental change in individuals with ASD (Raznahan et al. 2010; Scheel et al. 2011).

DTI measures of whole brain FA, MD, $\mathrm{AD}$, and RD also did not differ between groups. To date, few studies have examined alterations in white-matter fibre pathways in children with ASD. One older study reported in a sample of seven children and adolescents reduced FA in ASD in regions throughout the brain (Barnea-Goraly et al. 2004). Another study with a larger sample $(n=19)$ found greater changes in children compared to adolescents with ASD (Ameis et al. 2011). Age effects in DTI measures could be a focus of a future, larger study.

\section{Thalamic volumes in children with ASD}

Previous investigation of the volume of the entire thalamus in individuals with ASD identified a reduction in comparison to controls when controlling for total cerebral volume (Tamura et al. 2010; Tsatsanis et al. 2003), while other reports found no differences in thalamic volumes in their sample (Gaffney et al. 1989; Hardan et al. 2006a). In the current study, thalamic volumes were larger in the ASD than TD children. The differences in findings may have been related to the age of the participants, as the two previous studies included participants with a wide age range (8-45 years), while our age range was comparatively narrow (6-15 years). In combination with these prior findings, our data may add credence to the early overgrowth hypothesis in ASD where cortical and subcortical structures have an increase in neuronal number in childhood that then undergo a more rapid age-related loss (Courchesne 2001, 2003, 2004, 2011; Courchesne and Pierce 2005). Future cross-sectional or longitudinal studies are necessary to address the developmental time course of the thalamus in ASD.

Self-injury is associated with reduced thickness in cortical-somatosensory areas in children with ASD

Children who had high self-injury scores had decreased thickness in the right superior parietal lobe (SPL) and bilateral primary somatosensory cortices in comparison to children with low or no self-injury. The posterior parietal cortices mediate many aspects of somatosensation such as bodily spatial awareness, sensorimotor integration and attention, and also motor planning (Freund 2001). Lesions to the posterior parietal cortex result in deficits in sensorimotor integration and object-directed behaviours (Freund 2003; Wolpert et al. 1998). In relation to the current findings, repetitive injury may result in augmented somatosensory awareness such that proprioceptive and tactile stimuli are actively sought. In other sensory domains, children with ASD will scratch surfaces to induce sounds or seek/create multiple visual stimuli (e.g. crossing fingers in front of eyes) (Ornitz 1974). In a larger sample of children and adolescents with $\operatorname{ASD}(N=241)$, atypical sensory processing was the largest predictor of self-injury (Duerden et al. 2012) indicating that some parietal lobemediated processes may be altered in children with ASD who self-injure. Future studies focussing on the influence of altered sensory processing on self-injury in relation to parietal lobe function and structure are warranted.

Bilateral SI exhibited decreased cortical thickness in the children with higher self-injury scores. The location of the decrease in the left hemisphere was primarily in the hand region of SI, while the right-sided decrease was located in the leg area. Potentially, these findings may reflect possible somatopographical alterations in thickness as a result of self-injury. The neurons of SI contain small receptive fields that result in a fine somatotopic organization of body part representations (Kaas et al. 1979; Merzenich et al. 1978; Penfield and Boldrey 1937; Pons et al. 1985). The results of the current study may be an example of plasticity in the somatosensory system due to overuse as seen in individuals with writer's cramp (Delmaire et al. 2007). Alternatively, these changes may also be a result of repeated exposure to noxious insult. Repeated painful stimulation in healthy participants has resulted in somatotopically discrete changes in brain morphology in SI (Teutsch et al. 2008). Furthermore, hours of crossed-legged meditation practice, which can be perceived as painful, was associated with increased thickness in SI in the leg area (Grant et al. 2010). In the current study, the location of the injuries was not consistently localized on the body making it difficult to draw parallels with these other findings; another consideration is that due to the effects of smoothing the cortical thickness data $(20 \mathrm{~mm})$ and possible registration errors, the alterations in thickness may not be somatotopically specific. Much larger patient samples and individual analyses would be required to address these issues.

Self-injury is associated with reduced thalamic volumetry

Higher self-injury scores were associated with reduced volume of the VP nucleus of the thalamus, which may reflect a role for the thalamus in more general sensory interests and motor behaviour seen in children with ASD. An additional consideration is that this nucleus is the primary relay site for SI and somatosensory association 
cortices (Zhang et al. 2001). These results indicate that self-injurious behaviour may lead to structural changes localized to cortical and subcortical somatosensory processing regions.

Previous volumetric studies reported a lack of linear correlation between the volume of the thalamus and that of the entire cerebrum in individuals with ASD (Hardan et al. 2006a; Tsatsanis et al. 2003), which were interpreted to indicate a deficit or disruption in thalamocortical connections. In this previous work, self-injury was not specifically assessed and general stereotyped behaviours did not correlate with the volume of the thalamus. However, the volume of each thalamic nucleus was not considered in the analyses making it difficult to compare these results with that of the current study.

White-matter disturbances associated with self-injury

Children with high self-injury scores had lower FA and higher MD in the territory of the left PLIC. More severe self-injury scores were positively correlated with RD values in the bilateral PLIC and corona radiata. Given the findings in bilateral SI and VP nucleus, the alterations in these pathways may indicate a disruption in thalamocortical white-matter fibre pathways, which is more pronounced on the left (subserving the dominant hand in most cases).

Thakkar et al. (2008) examined the relation between repetitive behaviours and DTI measures in high-functioning adults with ASD. The authors reported decreased FA in the territory of the paracentral gyrus that extended into SI. However, these changes were not associated with displays of repetitive behaviours. The discrepancy in between their results and the current study may be due to the difference in age of the participants. Additionally, Thakkar et al. (2008) did not examine changes in other DTI measures such as individual eigenvalues (i.e. $\mathrm{AD}$ and $\mathrm{RD}$ ) that can provide more detailed information on the types of changes being observed in the white matter in individuals who self-injure.

In the present study, in addition to $\mathrm{FA}, \mathrm{RD}$, and $\mathrm{AD}$ were assessed in relation to self-injury scores. While the biological basis of increased RD still remains uncertain, its measure is reflective of the diffusion of water molecules running perpendicular to the axon sheath. Therefore, the increased RD values in self-injurers may be due to changes in the upregulation of ion channels leading to increased membrane permeability and a reduced axonal calibre. The increase in $\mathrm{RD}$ coupled with no change in $\mathrm{AD}$ values may indicate myelin loss in the children who self-injure, rather than a change in axonal number (Hüppi et al. 1998). For example, an animal model of dysmyelination showed increased $\mathrm{RD}$, not $\mathrm{AD}$, and was therefore believed to point towards myelin loss rather than the destruction of axons (Song et al. 2002).

\section{Conclusions}

Findings indicate that self-injury in children with ASD is associated with reduced grey matter in the right superior parietal lobe, a brain region involved in internal awareness, and also in bilateral SI, which processes tactile and nociceptive input. Additionally, these findings extended to reduced volume in the left VP nucleus, which sends somatosensory afferents to SI, as well as atypicalities in the thalamocortical projections involved in relaying somatosensation. This constellation of structural brain changes may be a result of use-dependent changes combined with noxious-stimulus induced alterations. While in the current study, it was not possible to determine whether altered pain reactivity was associated with alterations in the somatosensory system, this issue could be addressed in future work.

The actual causal relations between injury and brain structure and whether these effects would be seen only in individuals with ASD remain uncertain. Due to genetic or environmental factors, it is possible that children with ASD may have greater maturational abnormalities that lead to atypical structure of the somatosensory system. Future longitudinal studies with larger samples are needed to better understand this complex brain-behaviour association in this high-risk population.

Acknowledgments The authors would like to thank Wayne Lee for MRI technical and Dr. Annie Dupuis, Hospital for Sick Children, for statistical analysis support. This research was funded by the Canadian Institutes of Health Research [grant number MOP-81161 to MJT], Research Training Competition Fellowship from the Hospital for Sick Children (EGD), and a Reva Gerstein Fellowship in Paediatric Psychology (EGD). We also sincerely thank the children and their families who participated in this study.

\section{References}

Ameis SH, Fan J, Rockel C, Voineskos AN, Lobaugh NJ, Soorya L, Wang AT, Hollander E, Anagnostou E (2011) Impaired structural connectivity of socio-emotional circuits in autism spectrum disorders: a diffusion tensor imaging study. PLoS One 6(11):e28044

Armstrong D, Matt M (1999) Autoextraction in an autistic dental patient: a case report. Spec Care Dentist 19(2):72-74

Aylward EH, Minshew NJ, Field K, Sparks BF, Singh N (2002) Effects of age on brain volume and head circumference in autism. Neurology 59(2):175-183

Baghdadli A, Pascal C, Grisi S, Aussilloux C (2003) Risk factors for self-injurious behaviours among 222 young children with autistic disorders. J Intellect Disabil Res 47(Pt 8):622-627

Baranek GT (2002) Efficacy of sensory and motor interventions for children with autism. J Autism Dev Disord 32(5):397-422

Barnea-Goraly N, Kwon H, Menon V, Eliez S, Lotspeich L, Reiss AL (2004) White matter structure in autism: preliminary evidence from diffusion tensor imaging. Biol Psychiatry 55(3):323-326

Bodfish JW, Symonds FW, Parker DE, Lewis MH (1999) The Repetitive Behavior Scale. Western Carolina Center Research Reports 
Boucher M, Evans A, Siddiqi K (2009) Oriented morphometry of folds on surfaces. Inf Process Med Imaging 21:614-625

Carper RA, Courchesne E (2005) Localized enlargement of the frontal cortex in early autism. Biol Psychiatry 57(2):126-133

Carper RA, Moses P, Tigue ZD, Courchesne E (2002) Cerebral lobes in autism: early hyperplasia and abnormal age effects. Neuroimage 16(4): 1038-1051

Chakravarty MM, Bertrand G, Hodge CP, Sadikot AF, Collins DL (2006) The creation of a brain atlas for image guided neurosurgery using serial histological data. Neuroimage 30(2):359-376

Chakravarty MM, Sadikot AF, Germann J, Bertrand G, Collins DL (2008) Towards a validation of atlas warping techniques. Med Image Anal 12(6):713-726

Chakravarty MM, Sadikot AF, Germann J, Hellier P, Bertrand G, Collins DL (2009) Comparison of piece-wise linear, linear, and nonlinear atlas-to-patient warping techniques: analysis of the labeling of subcortical nuclei for functional neurosurgical applications. Hum Brain Mapp 30(11):3574-3595

Chang LC, Jones DK, Pierpaoli C (2005) RESTORE: robust estimation of tensors by outlier rejection. Magn Reson Med 53(5):1088-1095

Chung MK, Worsley KJ, Taylor J, Ramsay JO, Robbins S, Evans AC (2001) Diffusion smoothing on the cortical surface. Neuroimage 13S:95

Collins DL, Neelin P, Peters TM, Evans AC (1994) Automatic 3D intersubject registration of MR volumetric data in standardized Talairach space. J Comput Assist Tomogr 18(2):192-205

Cook PA, Bai Y, Nedjati-Gilani S, Seunarine KK, Hall MG, Parker GJ, Alexander DC (2006) Camino:open-source diffusion-MRI reconstruction and processing. 4th Scientific Meeting of the International Society for Magnetic Resonance in Medicine, Seattle, p 2759

Courchesne E (2004) Brain development in autism: early overgrowth followed by premature arrest of growth. Ment Retard Dev Disabil Res Rev 10(2):106-111

Courchesne E, Pierce K (2005) Brain overgrowth in autism during a critical time in development: implications for frontal pyramidal neuron and interneuron development and connectivity. Int J Dev Neurosci 23(2-3):153-170

Courchesne E, Karns CM, Davis HR, Ziccardi R, Carper RA, Tigue ZD, Chisum HJ, Moses P, Pierce K, Lord C, Lincoln AJ, Pizzo S, Schreibman L, Haas RH, Akshoomoff NA, Courchesne RY (2001) Unusual brain growth patterns in early life in patients with autistic disorder: an MRI study. Neurology 57(2):245-254

Courchesne E, Carper R, Akshoomoff N (2003) Evidence of brain overgrowth in the first year of life in autism. JAMA 290(3):337-344

Courchesne E, Campbell K, Solso S (2011) Brain growth across the life span in autism: age-specific changes in anatomical pathology. Brain Res 1380:138-145

Delmaire C, Vidailhet M, Elbaz A, Bourdain F, Bleton JP, Sangla S, Meunier S, Terrier A, Lehericy S (2007) Structural abnormalities in the cerebellum and sensorimotor circuit in writer's cramp. Neurology 69(4):376-380

Doyle-Thomas KA, Kushki A, Duerden EG, Taylor MJ, Lerch JP, Soorya LV, Wang AT, Fan J, Anagnostou E (2012) The effect of diagnosis, age, and symptom severity on cortical surface area in the cingulate cortex and insula in Autism spectrum disorders. J Child Neurol. doi:10.1177/0883073812451496

Duerden EG, Oatley HK, Mak-Fan KM, McGrath PA, Satzmari P, Roberts SW (2012) Risk factors associated with self-injurious behaviors in children and adolescents with autism spectrum disorders. J Autism Dev Disord 42(11):2460-2470

Freund HJ (2001) The parietal lobe as a sensorimotor interface: a perspective from clinical and neuroimaging data. Neuroimage 14(1 Pt 2):S142-S146
Freund HJ (2003) Somatosensory and motor disturbances in patients with parietal lobe lesions. Adv Neurol 93:179-193

Gaffney GR, Kuperman S, Tsai LY, Minchin S (1989) Forebrain structure in infantile autism. J Am Acad Child Adolesc Psychiatry 28(4):534-537

Grant JA, Courtemanche J, Duerden EG, Duncan GH, Rainville P (2010) Cortical thickness and pain sensitivity in zen meditators. Emotion 10(1):43-53

Hadjikhani N, Joseph RM, Snyder J, Tager-Flusberg H (2006) Anatomical differences in the mirror neuron system and social cognition network in autism. Cereb Cortex 16(9):1276-1282

Haller S, Borgwardt SJ, Schindler C, Aston J, Radue EW, RiecherRossler A (2009) Can cortical thickness asymmetry analysis contribute to detection of at-risk mental state and first-episode psychosis? a pilot study. Radiology 250(1):212-221

Hamilton LS, Narr KL, Luders E, Szeszko PR, Thompson PM, Bilder RM, Toga AW (2007) Asymmetries of cortical thickness: effects of handedness, sex, and schizophrenia. NeuroReport 18(14):1427-1431

Hardan AY, Minshew NJ, Mallikarjuhn M, Keshavan MS (2001) Brain volume in autism. J Child Neurol 16(6):421-424

Hardan AY, Jou RJ, Keshavan MS, Varma R, Minshew NJ (2004) Increased frontal cortical folding in autism: a preliminary MRI study. Psychiatry Res 131(3):263-268

Hardan AY, Girgis RR, Adams J, Gilbert AR, Keshavan MS, Minshew NJ (2006a) Abnormal brain size effect on the thalamus in autism. Psychiatry Res Neuroimaging 147(2-3):145-151

Hardan AY, Muddasani S, Vemulapalli M, Keshavan MS, Minshew NJ (2006b) An MRI study of increased cortical thickness in autism. Am J Psychiatry 163(7):1290-1292

Hildebrand C, Waxman SG (1984) Postnatal differentiation of rat optic nerve fibers: electron microscopic observations on the development of nodes of Ranvier and axoglial relations. J Comp Neurol 224(1):25-37

Hof PR, Knabe R, Bovier P, Bouras C (1991) Neuropathological observations in a case of autism presenting with self-injury behavior. Acta Neuropathol 82(4):321-326

Hüppi PS, Maier SE, Peled S, Zientara GP, Barnes PD, Jolesz FA, Volpe JJ (1998) Microstructural development of human newborn cerebral white matter assessed in vivo by diffusion tensor magnetic resonance imaging. Pediatr Research 44(4):584-590

Hyde KL, Samson F, Evans AC, Mottron L (2010) Neuroanatomical differences in brain areas implicated in perceptual and other core features of autism revealed by cortical thickness analysis and voxel-based morphometry. Hum Brain Mapp 31(4):556-566

Kaas J, Nelson R, Sur M, Lin C, Merzenich M (1979) Multiple representations of the body within the primary somatosensory cortex of primates. Science 204(4392):521-523

Lam KS, Aman MG (2007) The repetitive behavior scale-revised: independent validation in individuals with autism spectrum disorders. J Autism Dev Disord 37(5):855-866

Lax ID, Duerden EG, Lin SY, Mallar Chakravarty M, Donner EJ, Lerch JP, Taylor MJ (2013) Neuroanatomical consequences of very preterm birth in middle childhood. Brain Struct Funct 218(2):575-585

Le Couteur A, Lord C, Rutter M (2003) Autism diagnostic interviewrevised (ADI-R). Western Psychological Services, Los Angeles

Lerch JP, Pruessner JC, Zijdenbos A, Hampel H, Teipel SJ, Evans AC (2005) Focal decline of cortical thickness in Alzheimer's disease identified by computational neuroanatomy. Cereb Cortex 15(7):995-1001

Lipton M, Kim N, Park Y, Hulkower M, Gardin T, Shifteh K, Kim M, Zimmerman M, Lipton R, Branch C (2012) Robust detection of traumatic axonal injury in individual mild traumatic brain injury patients: intersubject variation, change over time and bidirectional changes in anisotropy. Brain Imaging Behav 6(2):329-342 
Lord C, Risi S, Lambrecht L, Cook EH Jr, Leventhal BL, DiLavore PC, Pickles A, Rutter M (2000) The autism diagnostic observation schedule-generic: a standard measure of social and communication deficits associated with the spectrum of autism. J Autism Dev Disord 30(3):205-223

Lyttelton O, Boucher M, Robbins S, Evans A (2007) An unbiased iterative group registration template for cortical surface analysis. Neuroimage 34(4):1535-1544

Mak-Fan K, Taylor M, Roberts W, Lerch J (2012) Measures of cortical grey matter structure and development in Children with autism spectrum disorder. J Autism Develop Disord 42(3): $419-427$

Medina AC, Sogbe R, Gomez-Rey AM, Mata M (2003) Factitial oral lesions in an autistic paediatric patient. Int $\mathrm{J}$ Paediatr Dent 13(2): 130-137

Merzenich MM, Kaas JH, Sur M, Lin C-S (1978) Double representation of the body surface within cytoarchitectonic area $3 \mathrm{~b}$ and 1 in "SI" in the owl monkey (aotus trivirgatus). J Comp Neurol 181(1):41-73

Militerni R, Bravaccio C, Falco C, Fico C, Palermo MT (2002) Repetitive behaviors in autistic disorder. Eur Child Adolesc Psychiatry 11(5):210-218

Misaki M, Wallace GL, Dankner N, Martin A, Bandettini PA (2012) Characteristic cortical thickness patterns in adolescents with autism spectrum disorders: interactions with age and intellectual ability revealed by canonical correlation analysis. Neuroimage 60(3):1890-1901

Nordahl CW, Dierker D, Mostafavi I, Schumann CM, Rivera SM, Amaral DG, Van Essen DC (2007) Cortical folding abnormalities in autism revealed by surface-based morphometry. J Neurosci 27(43):11725-11735

Ornitz EM (1974) The modulation of sensory input and motor output in autistic children. J Autism Child Schizophr 4(3):197-215

Penfield W, Boldrey E (1937) Somatic motor and sensory representation in the cerebral cortex of man as studied by electrical stimulation. Brain 60(4):389-443

Pons TP, Garraghty PE, Cusick CG, Kaas JH (1985) The somatotopic organization of area 2 in macaque monkeys. J Comp Neurol 241(4):445-466

Qiu D, Tan L-H, Zhou K, Khong P-L (2008) Diffusion tensor imaging of normal white matter maturation from late childhood to young adulthood: voxel-wise evaluation of mean diffusivity, fractional anisotropy, radial and axial diffusivities, and correlation with reading development. NeuroImage 41(2):223-232

Raznahan A, Toro R, Daly E, Robertson D, Murphy C, Deeley Q, Bolton PF, Paus T, Murphy DGM (2010) Cortical anatomy in autism spectrum disorder: an in vivo MRI study on the effect of age. Cereb Cortex 20(6):1332-1340

Ross-Russell M, Sloan P (2005) Autoextraction in a child with autistic spectrum disorder. Br Dent J 198(8):473-474

Scheel C, Rotarska-Jagiela A, Schilbach L, Lehnhardt FG, Krug B, Vogeley K, Tepest R (2011) Imaging derived cortical thickness reduction in high-functioning autism: key regions and temporal slope. Neuroimage 58(2):391-400

Sled JG, Zijdenbos AP, Evans AC (1998) A nonparametric method for automatic correction of intensity nonuniformity in MRI data. IEEE Trans Med Imaging 17(1):87-97

Smith SM (2002) Fast robust automated brain extraction. Hum Brain Mapp 17(3):143-155

Smith SM, Jenkinson M, Woolrich MW, Beckmann CF, Behrens TE, Johansen-Berg H, Bannister PR, De Luca M, Drobnjak I, Flitney DE, Niazy RK, Saunders J, Vickers J, Zhang Y, De Stefano N, Brady JM, Matthews PM (2004) Advances in functional and structural MR image analysis and implementation as FSL. NeuroImage 23(Suppl 1):S208-S219
Smith SM, Jenkinson M, Johansen-Berg H, Rueckert D, Nichols TE, Mackay CE, Watkins KE, Ciccarelli O, Cader MZ, Matthews PM, Behrens TE (2006) Tract-based spatial statistics: voxelwise analysis of multi-subject diffusion data. NeuroImage 31(4):1487-1505

Song SK, Sun SW, Ramsbottom MJ, Chang C, Russell J, Cross AH (2002) Dysmyelination revealed through MRI as increased radial (but unchanged axial) diffusion of water. NeuroImage 17(3):1429-1436

Suzuki Y, Matsuzawa H, Kwee IL, Nakada T (2003) Absolute eigenvalue diffusion tensor analysis for human brain maturation. NMR Biomed 16(5):257-260

Takahashi M, Ono J, Harada K, Maeda M, Hackney DB (2000) Diffusional anisotropy in cranial nerves with maturation: quantitative evaluation with diffusion MR imaging in rats. Radiology 216:881-885

Tamura R, Kitamura H, Endo T, Hasegawa N, Someya T (2010) Reduced thalamic volume observed across different subgroups of autism spectrum disorders. Psychiatry Res 184(3):186-188

Teutsch S, Herken W, Bingel U, Schoell E, May A (2008) Changes in brain gray matter due to repetitive painful stimulation. Neuroimage 42(2):845-849

Thakkar KN, Polli FE, Joseph RM, Tuch DS, Hadjikhani N, Barton JJ, Manoach DS (2008) Response monitoring, repetitive behaviour and anterior cingulate abnormalities in autism spectrum disorders (ASD). Brain 131(Pt 9):2464-2478

Tohka J, Zijdenbos A, Evans A (2004) Fast and robust parameter estimation for statistical partial volume models in brain MRI. Neuroimage 23(1):84-97

Tordjman S, Anderson GM, Botbol M, Brailly-Tabard S, Perez-Diaz F, Graignic R, Carlier M, Schmit G, Rolland AC, Bonnot O, Trabado S, Roubertoux P, Bronsard G (2009) Pain reactivity and plasma beta-endorphin in children and adolescents with autistic disorder. PLoS One 4(8):e5289

Tsatsanis KD, Rourke BP, Klin A, Volkmar FR, Cicchetti D, Schultz RT (2003) Reduced thalamic volume in high-functioning individuals with autism. Biol Psychiatry 53(2):121-129

Wallace GL, Dankner N, Kenworthy L, Giedd JN, Martin A (2010) Age-related temporal and parietal cortical thinning in autism spectrum disorders. Brain 133(Pt 12):3745-3754

Wegiel J, Kuchna I, Nowicki K, Imaki H, Marchi E, Ma SY, Chauhan A, Chauhan V, Bobrowicz TW, de Leon M, Louis LA, Cohen IL, London E, Brown WT, Wisniewski T (2010) The neuropathology of autism: defects of neurogenesis and neuronal migration, and dysplastic changes. Acta Neuropathol 119(6):755-770

Wolpert DM, Goodbody SJ, Husain M (1998) Maintaining internal representations: the role of the human superior parietal lobe. Nat Neurosci 1(6):529-533

Worsley KJ, Marrett S, Neelin P, Vandal AC, Friston KJ, Evans AC (1996) A unified statistical approach for determining significant signals in images of cerebral activation. Hum Brain Mapp 4(1):58-73

Worsley KJ, Taylor JE, Carbonell F, Chung MK, Duerden E, Bernhardt B, Lyttelton O, Boucher M, Evans AC (2009) SurfStat: a Matlab toolbox for the statistical analysis of univariate and multivariate surface and volumetric data using linear mixed effects models and random field theory. Proceedings of the15th Annual Meeting of the Organization for Human Brain Mapping. Neuroimage, San Francisco

Zhang HQ, Murray GM, Coleman GT, Turman AB, Zhang SP, Rowe MJ (2001) Functional characteristics of the parallel SI- and SIIprojecting neurons of the thalamic ventral posterior nucleus in the marmoset. J Neurophysiol 85(5):1805-1822

Zijdenbos AP, Forghani R, Evans AC (2002) Automatic "pipeline" analysis of 3-D MRI data for clinical trials: application to multiple sclerosis. IEEE Trans Med Imaging 21(10):1280-1291 